\title{
COMPLETE INTERSECTION VANISHING IDEALS ON SETS OF CLUTTER TYPE OVER FINITE FIELDS
}

\author{
AZUCENA TOCHIMANI AND RAFAEL H. VILLARREAL
}

\begin{abstract}
In this paper we give a classification of complete intersection vanishing ideals on parameterized sets of clutter type over finite fields.
\end{abstract}

\section{INTRODUCTION}

Let $R=K[\mathbf{y}]=K\left[y_{1}, \ldots, y_{n}\right]$ be a polynomial ring over a finite field $K=\mathbb{F}_{q}$ and let $y^{v_{1}}, \ldots, y^{v_{s}}$ be a finite set of monomials in $K[\mathbf{y}]$. As usual we denote the affine and projective spaces over the field $K$ of dimensions $s$ and $s-1$ by $\mathbb{A}^{s}$ and $\mathbb{P}^{s-1}$, respectively. Points of the projective space $\mathbb{P}^{s-1}$ are denoted by $[\alpha]$, where $0 \neq \alpha \in \mathbb{A}^{s}$.

We consider a set $\mathbb{X}$, in the projective space $\mathbb{P}^{s-1}$, parameterized by $y^{v_{1}}, \ldots, y^{v_{s}}$. The set $\mathbb{X}$ consists of all points $\left[\left(x^{v_{1}}, \ldots, x^{v_{s}}\right)\right]$ in $\mathbb{P}^{s-1}$ that are well defined, i.e., $x \in K^{n}$ and $x^{v_{i}} \neq 0$ for some $i$. The set $\mathbb{X}$ is called of clutter type if $\operatorname{supp}\left(y^{v_{i}}\right) \not \subset \operatorname{supp}\left(y^{v_{j}}\right)$ for $i \neq j$, where $\operatorname{supp}\left(y^{v_{i}}\right)$ is the support of the monomial $y^{v_{i}}$ consisting of the variables that occur in $y^{v_{i}}$. In this case we say that the set of monomials $y^{v_{1}}, \ldots, y^{v_{s}}$ is of clutter type. This terminology comes from the fact that the condition $\operatorname{supp}\left(y^{v_{i}}\right) \not \subset \operatorname{supp}\left(y^{v_{j}}\right)$ for $i \neq j$ means that there is a clutter $\mathcal{C}$, in the sense of [14], with vertex set $V(\mathcal{C})=\left\{y_{1}, \ldots, y_{n}\right\}$ and edge set

$$
E(\mathcal{C})=\left\{\operatorname{supp}\left(y^{v_{1}}\right), \ldots, \operatorname{supp}\left(y^{v_{s}}\right)\right\} .
$$

A clutter is also called a simple hypergraph, see Definition 2.8 .

Let $S=K\left[t_{1}, \ldots, t_{s}\right]=\oplus_{d=0}^{\infty} S_{d}$ be a polynomial ring over the field $K$ with the standard grading. The graded ideal $I(\mathbb{X})$ generated by the homogeneous polynomials of $S$ that vanish at all points of $\mathbb{X}$ is called the vanishing ideal of $\mathbb{X}$.

There are good reasons to study vanishing ideals over finite fields. They are used in algebraic coding theory [8] and in polynomial interpolation problems [5, 17]. The Reed-Muller-type codes arising from vanishing ideals on monomial parameterizations have received a lot of attention [1, 3, 6, 8, 10, 13, 14, 16].

The vanishing ideal $I(\mathbb{X})$ is a complete intersection if $I(\mathbb{X})$ is generated by $s-1$ homogeneous polynomials. Notice that $s-1$ is the height of $I(\mathbb{X})$ in the sense of [12]. The interest in complete intersection vanishing ideals over finite fields comes from information and communication theory, and algebraic coding theory [4, 7, 9].

Let $T$ be a projective torus in $\mathbb{P}^{s-1}$ (see Definition 2.15) and let $\mathbb{X}$ be the set in $\mathbb{P}^{s-1}$ parameterized by a clutter $\mathcal{C}$ (see Definition [2.9). Consider the set $X=\mathbb{X} \cap T$. In [14] it is shown that $I(X)$ is a complete intersection if and only if $X$ is a projective torus in $\mathbb{P}^{s-1}$. If the clutter

2000 Mathematics Subject Classification. Primary 14M10; Secondary 14G15, 13P25, 13P10, 11 T71.

Key words and phrases. Complete intersection, monomial parameterization, projective space, vanishing ideal, binomial ideal, finite field, Gröbner basis, clutter, Reed-Muller-type code.

The first author was partially supported by CONACyT. The second author was partially supported by SNI. 
$\mathcal{C}$ has all its edges of the same cardinality, in [15] a classification of the complete intersection property of $I(X)$ is given using linear algebra.

The main result of this paper is a classification of the complete intersection property of $I(\mathbb{X})$ when $\mathbb{X}$ is of clutter type (Theorem 2.19). Using the techniques of [13], this classification can be used to study the basic parameters [11, 19] of the Reed-Muller-type codes associated to $\mathbb{X}$.

For all unexplained terminology and additional information, we refer to [12] (for commutative algebra), 2] (for Gröbner bases), and [13, 17, 19] (for vanishing ideals and coding theory).

\section{Complete intersections}

In this section we give a full classification of the complete intersection property of vanishing ideals of sets of clutter type over finite fields. We continue to employ the notations and definitions used in Section 1.

Throughout this section $K=\mathbb{F}_{q}$ is a finite field, $y^{v_{1}}, \ldots, y^{v_{s}}$ are distinct monomials in the polynomial ring $R=K[\mathbf{y}]=K\left[y_{1}, \ldots, y_{n}\right]$, with $v_{i}=\left(v_{i 1}, \ldots, v_{i n}\right)$ and $y^{v_{i}}=y_{1}^{v_{i 1}} \cdots y_{n}^{v_{i n}}$ for $i=1, \ldots, s, \mathbb{X}$ is the set in $\mathbb{P}^{s-1}$ parameterized by these monomials, and $I(\mathbb{X})$ is the vanishing ideal of $\mathbb{X}$. Recall that $I(\mathbb{X})$ is the graded ideal of the polynomial ring $S=K\left[t_{1}, \ldots, t_{s}\right]$ generated by the homogeneous polynomials of $S$ that vanish on $\mathbb{X}$.

Definition 2.1. Given $a=\left(a_{1}, \ldots, a_{n}\right) \in \mathbb{N}^{n}$, we set $y^{a}:=y_{1}^{a_{1}} \cdots y_{n}^{a_{n}}$. The support of $y^{a}$, denoted $\operatorname{supp}\left(y^{a}\right)$, is the set of all $y_{i}$ such that $a_{i}>0$.

Definition 2.2. The set $\mathbb{X}$ is of clutter type if $\operatorname{supp}\left(y^{v_{i}}\right) \not \subset \operatorname{supp}\left(y^{v_{j}}\right)$ for $i \neq j$.

Definition 2.3. A binomial of $S$ is an element of the form $f=t^{a}-t^{b}$, for some $a, b$ in $\mathbb{N}^{s}$. An ideal generated by binomials is called a binomial ideal.

The set $\mathcal{S}=\mathbb{P}^{s-1} \cup\{[0]\}$ is a monoid under componentwise multiplication, that is, given $[\alpha]=\left[\left(\alpha_{1}, \ldots, \alpha_{s}\right)\right]$ and $[\beta]=\left[\left(\beta_{1}, \ldots, \beta_{s}\right)\right]$ in $\mathcal{S}$, the operation of this monoid is given by

$$
[\alpha] \cdot[\beta]=\left[\alpha_{1} \beta_{1}, \cdots, \alpha_{s} \beta_{s}\right],
$$

where $[\mathbf{1}]=[(1, \ldots, 1)]$ is the identity element.

Theorem 2.4. [18] If $K=\mathbb{F}_{q}$ is a finite field and $\mathbb{Y}$ is a subset of $\mathbb{P}^{s-1}$, then $I(\mathbb{Y})$ is a binomial ideal if and only if $\mathbb{Y} \cup\{[0]\}$ is a submonoid of $\mathbb{P}^{s-1} \cup\{[0]\}$.

Remark 2.5. Since $\mathbb{X}$ is parameterized by monomials, the set $\mathbb{X} \cup\{[0]\}$ is a monoid under componentwise multiplication. Hence, by Theorem $2.4, I(\mathbb{X})$ is a binomial ideal.

Lemma 2.6. Let $y^{v_{1}}, \ldots, y^{v_{s}}$ be a set of monomials such that $\operatorname{supp}\left(y^{v_{i}}\right) \not \subset \operatorname{supp}\left(y^{v_{j}}\right)$ for any $i \neq j$ and let $\mathcal{G}$ be a minimal generating set of $I(\mathbb{X})$ consisting of binomials. The following hold.

(a) If $0 \neq f=t_{j}^{a_{j}}-t^{c}$ for some $1 \leq j \leq s$ and some positive integer $a_{j}$, then $f \notin I(\mathbb{X})$.

(b) For each pair $1 \leq i<j \leq s$, there is $g_{i j}$ in $\mathcal{G}$ such that $g_{i j}= \pm\left(t_{i}^{c_{i j}} t_{j}-t^{b_{i j}}\right)$, where $c_{i j}$ is a positive integer less than or equal to $q$ and $b_{i j} \in \mathbb{N}^{s} \backslash\{0\}$.

(c) If $I(\mathbb{X})$ is a complete intersection, then $s \leq 4$.

Proof. (a): We proceed by contradiction. Assume that $f$ is in $I(\mathbb{X})$. Since $I(\mathbb{X})$ is a graded binomial ideal, the binomial $f$ is homogeneous of degree $a_{j}$, otherwise $t_{j}^{a_{j}}$ and $t^{c}$ would be in $I(\mathbb{X})$ which is impossible. Thus $c \in \mathbb{N}^{s} \backslash\{0\}$. Hence, as $f \neq 0$, we can pick $t_{i} \in \operatorname{supp}\left(t^{c}\right)$ with $i \neq j$. By hypothesis there is $y_{k} \in \operatorname{supp}\left(y^{v_{i}}\right) \backslash \operatorname{supp}\left(y^{v_{j}}\right)$, i.e., $v_{i k}>0$ and $v_{j k}=0$. Making $y_{k}=0$ and $y_{\ell}=1$ for $\ell \neq k$, we get that $f\left(y^{v_{1}}, \ldots, y^{v_{s}}\right)=1$, a contradiction. 
(b): The binomial $h=t_{i}^{q} t_{j}-t_{i} t_{j}^{q}$ vanishes at all points of $\mathbb{P}^{s-1}$, i.e., $h$ is in $I(\mathbb{X})$. Thus there is $g_{i j}$ in $\mathcal{G}$ such that $t_{i}^{q} t_{j}$ is a multiple of one of the two terms of the binomial $g_{i j}$. Hence, by part (a), the assertion follows.

(c): Since $I(\mathbb{X})$ is a complete intersection, there is a set of binomials $\mathcal{G}=\left\{g_{1}, \ldots, g_{s-1}\right\}$ that generate $I(\mathbb{X})$. The number of monomials that occur in $g_{1}, \ldots, g_{s-1}$ is at most $2(s-1)$. Thanks to part (b) for each pair $1 \leq i<j \leq s$, there is a monomial $t_{i}^{c_{i j}} t_{j}$, with $c_{i j} \in \mathbb{N}_{+}$, and a binomial $g_{i j}$ in $\mathcal{G}$ such that the monomial $t_{i}^{c_{i j}} t_{j}$ occurs in $g_{i j}$. As there are $s(s-1) / 2$ of these monomials, we get $s(s-1) / 2 \leq 2(s-1)$. Thus $s \leq 4$.

Lemma 2.7. Let $K$ be a field and let $I$ be the ideal of $S=K\left[t_{1}, t_{2}, t_{3}, t_{4}\right]$ generated by the binomials $g_{1}=t_{1} t_{2}-t_{3} t_{4}, g_{2}=t_{1} t_{3}-t_{2} t_{4}, g_{3}=t_{2} t_{3}-t_{1} t_{4}$. The following hold.

(i) $\mathcal{G}=\left\{t_{2} t_{3}-t_{1} t_{4}, t_{1} t_{3}-t_{2} t_{4}, t_{1} t_{2}-t_{3} t_{4}, t_{2}^{2} t_{4}-t_{3}^{2} t_{4}, t_{1}^{2} t_{4}-t_{3}^{2} t_{4}, t_{3}^{3} t_{4}-t_{3} t_{4}^{3}\right\}$ is a Gröbner basis of $I$ with respect to the GRevLex order $\prec$ on $S$.

(ii) If $\operatorname{char}(K)=2$, then $\operatorname{rad}(I) \neq I$.

(iii) If $\operatorname{char}(K) \neq 2$ and $e_{i}$ is the $i$-th unit vector, then $I=I(\mathbb{X})$, where

$$
\mathbb{X}=\left\{\left[e_{1}\right],\left[e_{2}\right],\left[e_{3}\right],\left[e_{4}\right],[(1,-1,-1,1)],[(1,1,1,1)],[(-1,-1,1,1)],[(-1,1,-1,1)]\right\} .
$$

Proof. (i): Using Buchberger's criterion [2, p. 84], it is seen that $\mathcal{G}$ is a Gröbner basis of $I$.

(ii): Setting $h=t_{1} t_{2}-t_{1} t_{3}$, we get $h^{2}=\left(t_{1} t_{2}\right)^{2}-\left(t_{1} t_{3}\right)^{2}=t_{1} t_{2} g_{1}+t_{1} t_{3} g_{2}$, where $g_{1}=t_{1} t_{2}-t_{3} t_{4}$ and $g_{2}=t_{1} t_{3}-t_{2} t_{4}$. Thus $h \in \operatorname{rad}(I)$. Using part (i) it is seen that $h \notin I$.

(iii): As $g_{i}$ vanishes at all points of $\mathbb{X}$ for $i=1,2,3$, we get the inclusion $I \subset I(\mathbb{X})$. Since $\mathbb{X} \cup\{0\}$ is a monoid under componentwise multiplication, by Theorem 2.4, $I(\mathbb{X})$ is a binomial ideal. Take a homogeneous binomial $f$ in $S$ that vanishes at all points of $\mathbb{X}$. Let $h=t^{a}-t^{b}$, $a=\left(a_{i}\right), b=\left(b_{i}\right)$, be the residue obtained by dividing $f$ by $\mathcal{G}$. Hence we can write $f=g+h$, where $g \in I$ and the terms $t^{a}$ and $t^{b}$ are not divisible by any of the leading terms of $\mathcal{G}$. It suffices to show that $h=0$. Assume that $h \neq 0$. As $h \in I(\mathbb{X})$ and $\left[e_{i}\right]$ is in $\mathbb{X}$ for all $i$, we get that $\left|\operatorname{supp}\left(t^{a}\right)\right| \geq 2$ and $\left|\operatorname{supp}\left(t^{b}\right)\right| \geq 2$. It follows that $h$ has one of the following forms:

$$
\begin{array}{lll}
h=t_{1} t_{4}^{i}-t_{2} t_{4}^{i}, & h=t_{1} t_{4}^{i}-t_{3} t_{4}^{i}, & h=t_{2} t_{4}^{i}-t_{3} t_{4}^{i}, \\
h=t_{3}^{2} t_{4}^{i-1}-t_{3} t_{4}^{i}, & h=t_{3}^{2} t_{4}^{i-1}-t_{2} t_{4}^{i}, & h=t_{3}^{2} t_{4}^{i-1}-t_{1} t_{4}^{i},
\end{array}
$$

where $i \geq 1$, a contradiction because none of these binomials vanishes at all points of $\mathbb{X}$.

Definition 2.8. A hypergraph $\mathcal{H}$ is a pair $(V(\mathcal{H}), E(\mathcal{H}))$ such that $V(\mathcal{H})$ is a finite set and $E(\mathcal{H})$ is a subset of the set of all subsets of $V(\mathcal{H})$. The elements of $E(\mathcal{H})$ and $V(\mathcal{H})$ are called edges and vertices, respectively. A hypergraph is simple if $f_{1} \not \subset f_{2}$ for any two edges $f_{1}, f_{2}$. A simple hypergraph is called a clutter and will be denoted by $\mathcal{C}$ instead of $\mathcal{H}$.

One example of a clutter is a graph with the vertices and edges defined in the usual way.

Definition 2.9. Let $\mathcal{C}$ be a clutter with vertex set $V(\mathcal{C})=\left\{y_{1}, \ldots, y_{n}\right\}$, let $f_{1}, \ldots, f_{s}$ be the edges of $\mathcal{C}$ and let $v_{k}=\sum_{x_{i} \in f_{k}} e_{i}$ be the characteristic vector of $f_{k}$ for $1 \leq k \leq s$, where $e_{i}$ is the $i$-th unit vector. The set in the projective space $\mathbb{P}^{s-1}$ parameterized by $y^{v_{1}}, \ldots, y^{v_{s}}$, denoted by $\mathbb{X}_{\mathcal{C}}$, is called the projective set parameterized by $\mathcal{C}$.

Lemma 2.10. Let $K=\mathbb{F}_{q}$ be a finite field with $q \neq 2$ elements, let $\mathcal{C}$ be a clutter with vertices $y_{1}, \ldots, y_{n}$, let $v_{1}, \ldots, v_{s}$ be the characteristic vectors of the edges of $\mathcal{C}$ and let $\mathbb{X}_{\mathcal{C}}$ be the projective set parameterized by $\mathcal{C}$. If $f=t_{i} t_{j}-t_{k} t_{\ell} \in I\left(\mathbb{X}_{\mathcal{C}}\right)$, with $i, j, k, l$ distinct, then $y^{v_{i}} y^{v_{j}}=y^{v_{k}} y^{v_{\ell}}$. 
Proof. For simplicity assume that $f=t_{1} t_{2}-t_{3} t_{4}$. Setting $A_{1}=\operatorname{supp}\left(y^{v_{1}} y^{v_{2}}\right), A_{2}=\operatorname{supp}\left(y^{v_{3}} y^{v_{4}}\right)$, $S_{1}=\operatorname{supp}\left(y^{v_{1}}\right) \cap \operatorname{supp}\left(y^{v_{2}}\right)$ and $S_{2}=\operatorname{supp}\left(y^{v_{3}}\right) \cap \operatorname{supp}\left(y^{v_{4}}\right)$, it suffices to show the equalities $A_{1}=A_{2}$ and $S_{1}=S_{2}$. If $A_{1} \not \subset A_{2}$, pick $y_{k} \in A_{1} \backslash A_{2}$. Making $y_{k}=0$ and $y_{\ell}=1$ for $\ell \neq k$, and using that $f$ vanishes on $\mathbb{X}_{\mathcal{C}}$, we get that $f\left(y^{v_{1}}, \ldots, y^{v_{4}}\right)=-1=0$, a contradiction. Thus $A_{1} \subset A_{2}$. The other inclusion follows by a similar reasoning. Next we show the equality $S_{1}=S_{2}$. If $S_{1} \not \subset S_{2}$, pick a variable $y_{k} \in S_{1} \backslash S_{2}$. Let $\beta$ be a generator of the cyclic group $\mathbb{F}_{q}^{*}=\mathbb{F}_{q} \backslash\{0\}$. Making $y_{k}=\beta, y_{\ell}=1$ for $\ell \neq k$, and using that $f$ vanishes on $\mathbb{X}_{\mathcal{C}}$ and the equality $A_{1}=A_{2}$, we get that $f\left(y^{v_{1}}, \ldots, y^{v_{4}}\right)=\beta^{2}-\beta=0$. Hence $\beta^{2}=\beta$ and $\beta=1$, a contradiction because $q \neq 2$. Thus $S_{1} \subset S_{2}$. The other inclusion follows by a similar argument.

Remark 2.11. Let $K=\mathbb{F}_{q}$ be a finite field with $q$ odd and let $\mathbb{X}$ be the set of clutter type in $\mathbb{P}^{3}$ parameterized by the following monomials:

$$
\begin{aligned}
& y^{v_{1}}=y_{1}^{q-1} y_{2}^{r} y_{3}^{r} y_{4}^{q-1} y_{5}^{q-1} y_{6}^{q-1} y_{7}^{q-1}, \\
& y^{v_{2}}=y_{1}^{r} y_{2}^{r} y_{3}^{q-1} y_{4}^{q-1} y_{5}^{q-1} y_{6}^{q-1} y_{8}^{q-1}, \\
& y^{v_{3}}=y_{2}^{q-1} y_{4}^{q-1} y_{1}^{r} y_{3}^{r} y_{5}^{q-1} y_{7}^{q-1} y_{8}^{q-1}, \\
& y^{v_{4}}=y_{1}^{q-1} y_{2}^{q-1} y_{3}^{q-1} y_{4}^{q-1} y_{6}^{q-1} y_{7}^{q-1} y_{8}^{q-1},
\end{aligned}
$$

where $r=(q-1) / 2$. Then

$$
\mathbb{X}=\left\{\left[e_{1}\right],\left[e_{2}\right],\left[e_{3}\right],\left[e_{4}\right],[(1,-1,-1,1)],[(1,1,1,1)],[(-1,-1,1,1)],[(-1,1,-1,1)]\right\},
$$

$|\mathbb{X}|=8$ and $I(\mathbb{X})=\left(t_{1} t_{2}-t_{3} t_{4}, t_{1} t_{3}-t_{2} t_{4}, t_{2} t_{3}-t_{1} t_{4}\right)$.

Below we show that the set $\mathbb{X}$ of Remark 2.11 cannot be parameterized by a clutter.

Remark 2.12. Let $K=\mathbb{F}_{q}$ be a field with $q \neq 2$ elements. Then the ideal

$$
I=\left(t_{1} t_{2}-t_{3} t_{4}, t_{1} t_{3}-t_{2} t_{4}, t_{2} t_{3}-t_{1} t_{4}\right)
$$

cannot be the vanishing ideal of a set in $\mathbb{P}^{3}$ parameterized by a clutter. Indeed assume that there is a clutter $\mathcal{C}$ such that $I=I\left(\mathbb{X}_{\mathcal{C}}\right)$ and $\mathbb{X}_{\mathcal{C}} \subset \mathbb{P}^{3}$. If $v_{1}, \ldots, v_{4}$ are the characteristic vectors of the edges of $\mathcal{C}$. Then, by Lemma 2.10, we get $v_{1}+v_{2}=v_{3}+v_{4}, v_{1}+v_{3}=v_{2}+v_{4}$ and $v_{2}+v_{3}=v_{1}+v_{4}$. It follows that $v_{1}=v_{2}=v_{3}=v_{4}$, a contradiction.

Lemma 2.13. Let $K$ be a field and let $I$ be the ideal of $S=K\left[t_{1}, t_{2}, t_{3}\right]$ generated by the binomials $g_{1}=t_{1} t_{2}-t_{2} t_{3}, g_{2}=t_{1} t_{3}-t_{2} t_{3}$. The following hold.

(i) $\mathcal{G}=\left\{t_{1} t_{3}-t_{2} t_{3}, t_{1} t_{2}-t_{2} t_{3}, t_{2}^{2} t_{3}-t_{2} t_{3}^{2}\right\}$ is a Gröbner basis of $I$ with respect to the GRevLex order $\prec$ on $S$.

(ii) $I=I(\mathbb{X})$, where $\mathbb{X}=\left\{\left[e_{1}\right],\left[e_{2}\right],\left[e_{3}\right],[(1,1,1)]\right\}$.

Proof. It follows using the arguments given in Lemma 2.7.

Remark 2.14. Let $K=\mathbb{F}_{q}$ be a finite field with $q$ elements and let $\mathbb{X}$ be the projective set in $\mathbb{P}^{2}$ parameterized by the following monomials:

$$
y^{v_{1}}=y_{1}^{q-1} y_{2}^{q-1}, y^{v_{2}}=y_{2}^{q-1} y_{3}^{q-1}, y^{v_{3}}=y_{1}^{q-1} y_{3}^{q-1} .
$$

Then $\mathbb{X}=\left\{\left[e_{1}\right],\left[e_{2}\right],\left[e_{3}\right],[(1,1,1)]\right\}$ and $I(\mathbb{X})=\left(t_{1} t_{2}-t_{2} t_{3}, t_{1} t_{3}-t_{2} t_{3}\right)$.

Definition 2.15. The set $T=\left\{\left[\left(x_{1}, \ldots, x_{s}\right)\right] \in \mathbb{P}^{s-1} \mid x_{i} \in K^{*}\right.$ for all $\left.i\right\}$ is called a projective torus in $\mathbb{P}^{s-1}$.

Lemma 2.16. Let $\beta$ be a generator of $\mathbb{F}_{q}^{*}$ and $0 \neq r \in \mathbb{N}$. Suppose $s=2$. If $I=\left(t_{1}^{r+1} t_{2}-t_{1} t_{2}^{r+1}\right)$ and $r$ divides $q-1$, then $I=I(\mathbb{X})$, where $\mathbb{X}$ is the set of clutter type in $\mathbb{P}^{1}$ parameterized by $y_{1}^{q-1}, y_{2}^{q-1} y_{3}^{k}$ and $r=\mathrm{o}\left(\beta^{k}\right)$. 
Proof. We set $f=t_{1}^{r+1} t_{2}-t_{1} t_{2}^{r+1}$. Take a point $P=\left[\left(x_{1}^{q-1}, x_{2}^{q-1} x_{3}^{k}\right)\right]$ in $\mathbb{X}$. Then

$$
f(P)=\left(x_{1}^{q-1}\right)^{r+1}\left(x_{2}^{q-1} x_{3}^{k}\right)-\left(x_{1}^{q-1}\right)\left(x_{2}^{q-1} x_{3}^{k}\right)^{r+1} .
$$

We may assume $x_{1} \neq 0$ and $x_{2} \neq 0$. Then $f(P)=x_{3}^{k}-\left(x_{3}^{k}\right)^{r+1}$. If $x_{3} \neq 0$, then $x_{3}=\beta^{i}$ for some $i$ and $\left(x_{3}^{k}\right)^{r+1}=x_{3}^{k}$, that is, $f(P)=0$. Therefore one has the inclusion $(f) \subset I(\mathbb{X})$.

Next we show the inclusion $I(\mathbb{X}) \subset(f)$. By Theorem 2.4, $I(\mathbb{X})$ is a binomial ideal. Take a non-zero binomial $g=t_{1}^{a_{1}} t_{2}^{a_{2}}-t_{1}^{b_{1}} t_{2}^{b_{2}}$ that vanishes on $\mathbb{X}$. Then $a_{1}+a_{2}=b_{1}+b_{2}$ because $I(\mathbb{X})$ is graded. We may assume that $b_{1}>a_{1}$ and $a_{2}>b_{2}$. We may also assume that $a_{1}>0$ and $b_{2}>0$ because $\left\{\left[e_{1}\right],\left[e_{2}\right]\right\} \subset \mathbb{X}$. Then $g=t_{1}^{a_{1}} t_{2}^{b_{2}}\left(t_{2}^{a_{2}-b_{2}}-t_{1}^{b_{1}-a_{1}}\right)$. As $g$ vanishes on $\mathbb{X}$, making $y_{3}=\beta$ and $y_{1}=y_{2}=1$, we get $\left(\beta^{k}\right)^{a_{2}-b_{2}}=1$. Hence $a_{2}-b_{2}=\lambda r$ for some $\lambda \in \mathbb{N}_{+}$, where $r=o\left(\beta^{k}\right)$. Thus $t_{2}^{a_{2}-b_{2}}-t_{1}^{b_{1}-a_{1}}$ is equal to $t_{2}^{\lambda r}-t_{1}^{\lambda r} \in\left(t_{1}^{r}-t_{2}^{r}\right)$. Therefore $g$ is a multiple of $f=t_{1} t_{2}\left(t_{1}^{r}-t_{2}^{r}\right)$ because $a_{1}>0$ and $b_{2}>0$. Thus $g \in(f)$.

Lemma 2.17. Let $K=\mathbb{F}_{q}$ be a finite field. If $\left\{\left[e_{1}\right],\left[e_{2}\right]\right\} \subset \mathbb{Y} \subset \mathbb{P}^{1}$ and $\mathbb{Y} \cup\{0\}$ is a monoid under componentwise multiplication, then there is $0 \neq r \in \mathbb{N}$ such that $I(\mathbb{Y})=\left(t_{1}^{r+1} t_{2}-t_{1} t_{2}^{r+1}\right)$ and $r$ divides $q-1$.

Proof. We set $f=t_{1}^{r+1} t_{2}-t_{1} t_{2}^{r+1}$ and $X=\mathbb{Y} \cap T$, where $T$ is a projective torus in $\mathbb{P}^{1}$. The set $X$ is a group, under componentwise multiplication, because $X$ is a finite monoid and the cancellation laws hold. By Theorem 2.4, $I(\mathbb{Y})$ is a binomial ideal. Clearly $(f) \subset I(\mathbb{Y})$. To show the other inclusion take a non-zero binomial $g=t_{1}^{a_{1}} t_{2}^{a_{2}}-t_{1}^{b_{1}} t_{2}^{b_{2}}$ that vanish on $\mathbb{Y}$. Then $a_{1}+a_{2}=b_{1}+b_{2}$ because $I(\mathbb{Y})$ is graded. We may assume that $b_{1}>a_{1}$ and $a_{2}>b_{2}$. We may also assume that $a_{1}>0$ and $b_{2}>0$ because $\left\{\left[e_{1}\right],\left[e_{2}\right]\right\} \subset \mathbb{X}$. Then $g=t_{1}^{a_{1}} t_{2}^{b_{2}}\left(t_{2}^{a_{2}-b_{2}}-t_{1}^{b_{1}-a_{1}}\right)$. The subgroup of $\mathbb{F}_{q}^{*}$ given by $H=\left\{\xi \in \mathbb{F}_{q}^{*} \mid[(1, \xi)] \in X\right\}$ has order $r=|X|$. Pick a generator $\beta$ of the cyclic group $\mathbb{F}_{q}^{*}$. Then $H$ is a cyclic group generated by $\beta^{k}$ for some $k \geq 0$. As $g$ vanishes on $\mathbb{Y}$, one has that $t_{2}^{a_{2}-b_{2}}-t_{1}^{b_{1}-a_{1}}$ vanishes on $X$. In particular $\left(\beta^{k}\right)^{a_{2}-b_{2}}=1$. Hence $a_{2}-b_{2}=\lambda r$ for some $\lambda \in \mathbb{N}_{+}$, where $r=o\left(\beta^{k}\right)=|X|$. Proceeding as in the proof of Lemma 2.16 one derives that $g \in(f)$. Noticing that $T$ has order $q-1$, we obtain that $r$ divides $q-1$.

Definition 2.18. An ideal $I \subset S$ is called a complete intersection if there exists $g_{1}, \ldots, g_{r}$ in $S$ such that $I=\left(g_{1}, \ldots, g_{r}\right)$, where $r$ is the height of $I$.

Recall that a graded ideal $I$ is a complete intersection if and only if $I$ is generated by a homogeneous regular sequence with ht $(I)$ elements (see [20, Proposition 2.3.19, Lemma 2.3.20]).

Theorem 2.19. Let $K=\mathbb{F}_{q}$ be a finite field and let $\mathbb{X}$ be a set in $\mathbb{P}^{s-1}$ parameterized by a set of monomials $y^{v_{1}}, \ldots, y^{v_{s}}$ such that $\operatorname{supp}\left(y^{v_{i}}\right) \not \subset \operatorname{supp}\left(y^{v_{j}}\right)$ for any $i \neq j$. Then $I(\mathbb{X})$ is a complete intersection if and only if $s \leq 4$ and, up to permutation of variables, $I(\mathbb{X})$ has one of the following forms:

(i) $s=4, q$ is odd and $I=\left(t_{1} t_{2}-t_{3} t_{4}, t_{1} t_{3}-t_{2} t_{4}, t_{2} t_{3}-t_{1} t_{4}\right)$.

(ii) $s=3$ and $I=\left(t_{1} t_{2}-t_{2} t_{3}, t_{1} t_{3}-t_{2} t_{3}\right)$.

(iii) $s=2$ and $I=\left(t_{1}^{r+1} t_{2}-t_{1} t_{2}^{r+1}\right)$, where $0 \neq r \in \mathbb{N}$ is a divisor of $q-1$.

(iv) $s=1$ and $I=(0)$.

Proof. $\Rightarrow$ ): Assume that $I(\mathbb{X})$ is a complete intersection. By Lemma 2.6(c) one has $s \leq 4$.

Case (i): Assume that $s=4$. Setting $I=I(\mathbb{X})$, by hypothesis $I$ is generated by 3 binomials $g_{1}, g_{2}, g_{3}$. By Lemma 2.6(b) for each pair $1 \leq i<j \leq 4$ there are positive integers $c_{i j}$ and $a_{i j}$ such that $t_{i}^{c_{i j}} t_{j}$ and $t_{i} t_{j}^{a_{i j}}$ occur as terms in $g_{1}, g_{2}, g_{3}$. Since there are at most 6 monomials that 
occur in the $g_{i}$ 's, we get that $c_{i j}=a_{i j}=1$ for $1 \leq i<j \leq 4$. Thus, up to permutation of variables, there are 4 subcases to consider:

$$
\begin{array}{llll}
(a): & g_{1}=t_{1}\left(t_{2}-t_{3}\right), & g_{2}=t_{1} t_{4}-t_{2} t_{3}, & g_{3}=t_{4}\left(t_{2}-t_{3}\right) . \\
(b): & g_{1}=t_{1}\left(t_{2}-t_{3}\right), & g_{2}=t_{4}\left(t_{1}-t_{3}\right), & g_{3}=t_{2}\left(t_{3}-t_{4}\right) . \\
(c): & g_{1}=t_{1} t_{2}-t_{3} t_{4}, & g_{2}=t_{1} t_{3}-t_{2} t_{4}, & g_{3}=t_{2} t_{3}-t_{1} t_{4} . \\
(d): & g_{1}=t_{3}\left(t_{1}-t_{2}\right), & g_{2}=t_{1}\left(t_{3}-t_{4}\right), & g_{3}=t_{2}\left(t_{1}-t_{4}\right) .
\end{array}
$$

Subcase (a): This case cannot occur because the ideal $\left(g_{1}, g_{2}, g_{3}\right)$ has height 2 .

Subcase (b): The reduced Gröbner basis of $I=\left(g_{1}, g_{2}, g_{3}\right)$ with respect to the GRevLex order $\prec$ is given by

$$
\begin{array}{lll}
g_{1}=t_{1} t_{2}-t_{1} t_{3}, & g_{2}=t_{1} t_{4}-t_{3} t_{4}, \quad g_{3}=t_{2} t_{3}-t_{2} t_{4}, \\
g_{4}=t_{3}^{2} t_{4}-t_{2} t_{4}^{2}, & g_{5}=t_{1} t_{3}^{2}-t_{2} t_{4}^{2}, \quad g_{6}=t_{2}^{2} t_{4}^{2}-t_{2} t_{4}^{3} .
\end{array}
$$

Hence the binomial $h=t_{2} t_{4}-t_{3} t_{4} \notin I$ because $t_{2} t_{4}$ does not belong to $\operatorname{in}_{\prec}(I)$, the initial ideal of $I$. Since $h^{2}=-2 t_{4}^{2} g_{3}+t_{4} g_{4}+g_{6}$, we get that $h \in \operatorname{rad}(I)$. Thus $I$ is not a radical ideal which is impossible because $I=I(\mathbb{X})$ is a vanishing ideal. Therefore this case cannot occur.

Subcase (c): In this case one has $I=\left(t_{1} t_{2}-t_{3} t_{4}, t_{1} t_{3}-t_{2} t_{4}, t_{2} t_{3}-t_{1} t_{4}\right)$, as required. From Lemma 2.7, we obtain that $q$ is odd.

Subcase (d): The reduced Gröbner basis of $I=\left(g_{1}, g_{2}, g_{3}\right)$ with respect to the GRevLex order $\prec$ is given by

$$
\begin{array}{ll}
h_{1}=t_{2} t_{3}-t_{1} t_{4}, & g_{2}=t_{1} t_{3}-t_{1} t_{4}, \quad g_{3}=t_{1} t_{2}-t_{2} t_{4}, \\
g_{4}=t_{1} t_{4}^{2}-t_{2} t_{4}^{2}, & g_{5}=t_{1}^{2} t_{4}-t_{2} t_{4}^{2}, \quad g_{6}=t_{2}^{2} t_{4}^{2}-t_{2} t_{4}^{3} .
\end{array}
$$

Setting $h=t_{1} t_{4}-t_{2} t_{4}$, as in Subcase (b), one can readily verify that $h \notin I$ and $h^{2} \in I$. Hence $I$ is not a radical ideal. Therefore this case cannot occur.

Case (ii): Assume that $s=3$. By hypothesis $I=I(\mathbb{X})$ is generated by 2 binomials $g_{1}, g_{2}$. By Lemma 2.6(b) for each pair $1 \leq i<j \leq 3$ there are positive integers $c_{i j}$ and $a_{i j}$ such that $t_{i}^{c_{i j}} t_{j}$ and $t_{i} t_{j}^{a_{i j}}$ occur as terms in $g_{1}, g_{2}$. Since there are at most 4 monomials that occur in the $g_{i}$ 's it is seen that, up to permutation of variables, there are 2 subcases to consider:

$$
\begin{array}{ll}
\text { (a) : } & g_{1}=t_{1} t_{3}-t_{2} t_{3}, g_{2}=t_{1}^{c_{12}} t_{2}-t_{1} t_{2}^{a_{12}} \text { with } c_{12}=a_{12} \geq 2 . \\
(b): & g_{1}=t_{1} t_{2}-t_{2} t_{3}, g_{2}=t_{1} t_{3}-t_{2} t_{3} .
\end{array}
$$

Subcase (a) cannot occur because the ideal $I=\left(g_{1}, g_{2}\right)$, being contained in $\left(t_{1}-t_{2}\right)$, has height 1. Thus we are left with subcase (b), that is, $I=\left(t_{1} t_{2}-t_{2} t_{3}, t_{1} t_{3}-t_{2} t_{3}\right)$, as required.

Case (iii): If $s=2$, then $\mathbb{X}$ is parameterized by $y^{v_{1}}, y^{v_{2}}$. Pick $y_{k} \in \operatorname{supp}\left(y^{v_{1}}\right) \backslash \operatorname{supp}\left(y^{v_{2}}\right)$. Making $y_{k}=0$ and $y_{\ell}=1$ for $\ell \neq k$, we get that $\left[e_{2}\right] \in \mathbb{X}$, and by a similar argument $\left[e_{1}\right] \in \mathbb{X}$. As $\mathbb{X} \cup\{[0]\}$ is a monoid under componentwise multiplication, by Lemma 2.17, $I(\mathbb{X})$ has the required form.

Case (iv): If $s=1$, this case is clear.

$\Leftarrow)$ The converse is clear because the vanishing ideal $I(\mathbb{X})$ has height $s-1$.

Proposition 2.20. If I is an ideal of $S$ of one of the following forms:

(i) $s=4, q$ is odd and $I=\left(t_{1} t_{2}-t_{3} t_{4}, t_{1} t_{3}-t_{2} t_{4}, t_{2} t_{3}-t_{1} t_{4}\right)$,

(ii) $s=3$ and $I=\left(t_{1} t_{2}-t_{2} t_{3}, t_{1} t_{3}-t_{2} t_{3}\right)$,

(iii) $s=2$ and $I=\left(t_{1}^{r+1} t_{2}-t_{1} t_{2}^{r+1}\right)$, where $0 \neq r \in \mathbb{N}$ and $r$ divides $q-1$,

then there is a set $\mathbb{X}$ in $\mathbb{P}^{s-1}$ of clutter type such that $I$ is the vanishing ideal $I(\mathbb{X})$. 
Proof. The result follows from Lemma 2.7 and Remark 2.11, Lemma 2.13 and Remark 2.14, and Lemma 2.16, respectively

Problem 2.21. Let $\mathbb{X}$ be a set of clutter type such that $I(\mathbb{X})$ is a complete intersection. Using the techniques of [4, 10, 13, 14, and Theorem 2.19 find formulas for the basic parameters of the Reed-Muller-type codes associated to $\mathbb{X}$.

\section{REFERENCES}

[1] C. Carvalho, On the second Hamming weight of some Reed-Muller type codes, Finite Fields Appl. 24 (2013), 88-94.

[2] D. Cox, J. Little and D. O'Shea, Ideals, Varieties, and Algorithms, Springer-Verlag, 1992.

[3] P. Delsarte, J. M. Goethals and F. J. MacWilliams, On generalized Reed-Muller codes and their relatives, Information and Control 16 (1970), 403-442.

[4] I. M. Duursma, C. Rentería and H. Tapia-Recillas, Reed-Muller codes on complete intersections, Appl. Algebra Engrg. Comm. Comput. 11 (2001), no. 6, 455-462.

[5] M. Gasca, Mariano and T. Sauer, Polynomial interpolation in several variables, Adv. Comput. Math. 12 (2000), no. 4, 377-410.

[6] O. Geil and C. Thomsen, Weighted Reed-Muller codes revisited, Des. Codes Cryptogr. 66 (2013), 195-220.

[7] L. Gold, J. Little and H. Schenck, Cayley-Bacharach and evaluation codes on complete intersections, J. Pure Appl. Algebra 196 (2005), no. 1, 91-99.

[8] M. González-Sarabia, C. Rentería and H. Tapia-Recillas, Reed-Muller-type codes over the Segre variety, Finite Fields Appl. 8 (2002), no. 4, 511-518.

[9] J. Hansen, Linkage and codes on complete intersections, Appl. Algebra Engrg. Comm. Comput. 14 (2003), no. $3,175-185$.

[10] H. H. López, C. Rentería and R. H. Villarreal, Affine cartesian codes, Des. Codes Cryptogr. 71 (2014), no. $1,5-19$.

[11] F.J. MacWilliams and N.J.A. Sloane, The Theory of Error-correcting Codes, North-Holland, 1977.

[12] H. Matsumura, Commutative Ring Theory, Cambridge Studies in Advanced Mathematics 8, Cambridge University Press, 1986.

[13] C. Rentería, A. Simis and R. H. Villarreal, Algebraic methods for parameterized codes and invariants of vanishing ideals over finite fields, Finite Fields Appl. 17 (2011), no. 1, 81-104.

[14] E. Sarmiento, M. Vaz Pinto and R. H. Villarreal, The minimum distance of parameterized codes on projective tori, Appl. Algebra Engrg. Comm. Comput. 22 (2011), no. 4, 249-264.

[15] E. Sarmiento, M. Vaz Pinto and R. H. Villarreal, On the vanishing ideal of an algebraic toric set and its parameterized linear codes, J. Algebra Appl. 11 (2012), no. 4, 1250072 (16 pages).

[16] A. Sørensen, Projective Reed-Muller codes, IEEE Trans. Inform. Theory 37 (1991), no. 6, 1567-1576.

[17] A. Tochimani and R. H. Villarreal, Vanishing ideals over rational parameterizations. Preprint, 2015, arXiv: $1502.05451 \mathrm{v} 1$.

[18] A. Tochimani and R. H. Villarreal, Vanishing ideals generated by binomials, preprint, 2015.

[19] M. Tsfasman, S. Vladut and D. Nogin, Algebraic geometric codes: basic notions, Mathematical Surveys and Monographs 139, American Mathematical Society, Providence, RI, 2007.

[20] R. H. Villarreal, Monomial Algebras, Second Edition, Monographs and Research Notes in Mathematics, Chapman and Hall/CRC, 2015.

Departamento de Matemáticas, Centro de Investigación y de Estudios Avanzados del IPN, Apartado Postal 14-740, 07000 Mexico City, D.F.

E-mail address: tochimani@math.cinvestav.mx

Departamento de Matemáticas, Centro de Investigación y de Estudios Avanzados del IPN, Apartado Postal 14-740, 07000 Mexico City, D.F.

E-mail address: vila@math.cinvestav.mx 